\title{
CONVERGENCE RATES FOR ESTIMATORS OF GEODESIC DISTANCES AND FRÈCHET EXPECTATIONS
}

\author{
CATHERINE AARON, ${ }^{*}$ Université Clermont Auvergne \\ OLIVIER BODART, ${ }^{* *}$ Université Jean Monnet
}

\begin{abstract}
Consider a sample $X_{n}=\left\{X_{1}, \ldots, X_{n}\right\}$ of independent and identically distributed variables drawn with a probability distribution $\mathbb{P}_{X}$ supported on a compact set $M \subset \mathbb{R}^{d}$. In this paper we mainly deal with the study of a natural estimator for the geodesic distance on $M$. Under rather general geometric assumptions on $M$, we prove a general convergence result. Assuming $M$ to be a compact manifold of known dimension $d^{\prime} \leq d$, and under regularity assumptions on $\mathbb{P}_{X}$, we give an explicit convergence rate. In the case when $M$ has no boundary, knowledge of the dimension $d^{\prime}$ is not needed to obtain this convergence rate. The second part of the work consists in building an estimator for the Fréchet expectations on $M$, and proving its convergence under regularity conditions, applying the previous results.
\end{abstract}

Keywords: Geometric inference; geodesic distance; statistics on manifolds; Fréchet expectations

2010 Mathematics Subject Classification: Primary 62-07

Secondary 62G05; 62G20; 62H99

\section{Introduction}

Let $\mathbb{P}_{X}$ be a probability distribution supported on a compact set $M \subset \mathbb{R}^{d}, d \geq 2$; that is, $M$ is the smallest closed set in $\mathbb{R}^{d}$ of probability 1 . Let $X_{n}=\left\{X_{1}, \ldots, X_{n}\right\}$ be a sample of independent and identically distributed (i.i.d) variables drawn on $M$ with the distribution $\mathbb{P}_{X}$. Our first aim is the study of a rather classical estimator of the geodesic distance on the unknown set $M$.

The way to build this estimator is quite intuitive (see, for example, [18]): given $r>0$, build a graph interconnecting all the pairs $\left(X_{i}, X_{j}\right)$ of the sample $X_{n}$ such that $\left\|X_{i}-X_{j}\right\| \leq r$. The geodesic distance between any two points $X_{k}$ and $X_{l}$ of the sample is then estimated by the length of the shortest path connecting $X_{k}$ and $X_{l}$ in the graph (see Definition 2.1 for details). This path (and its length) can be computed with optimal complexity by using Dijkstra's algorithm (see, for example, [3] for a presentation of this algorithm). As usual in such problems, $r=r_{n}$ must be a conveniently chosen sequence. First, it must converge to 0 as $n \rightarrow \infty$. Moreover, this convergence has to be slow enough for the path realizing the estimator to be smooth enough.

Received 20 July 2017; revision received 9 November 2018.

* Postal address: Laboratoire de Mathématiques Blaise Pascal (UMR 6620 - CNRS), Université Clermont Auvergne (Clermont-Ferrand 2), 63177 Aubière cedex, France. Email address: catherine.aaron@uca.fr

** Postal address: Institut Camille Jordan Faculté des Sciences et Techniques, Université Jean Monnet, 42023 SaintÉtienne Cedex 2, France. 
To the best of the authors' knowledge, the asymptotic behavior of such an estimator has not been studied yet. In a similar framework, the estimator

$$
\widehat{L}_{p}\left(X_{i}, X_{j}\right)=\min \left\{\sum_{k=1}^{K}\left\|X_{i_{k+1}}-X_{i_{k}}\right\|^{p}, i_{1}=i, i_{K}=j\right\}, \quad p \geq 1,
$$

was studied in [8], generalizing the results in [7] when $M$ is a compact manifold without boundary. The minimum was computed on all the paths in the graph connecting $X_{i}$ to $X_{j}$. The estimator studied in this paper roughly reads as follows (see Definition 2.1):

$$
\min \left\{\sum_{k=1}^{K}\left\|X_{i_{k+1}}-X_{i_{k}}\right\|, i_{1}=i, i_{K}=j \text { for all } k \text { such that }\left\|X_{i_{k+1}}-X_{i_{k}}\right\| \leq r_{n}\right\},
$$

where $\left(r_{n}\right)$ is a conveniently chosen sequence converging to 0 as $n \rightarrow \infty$. Here the minimum is then computed over the paths whose vertices satisfy a proximity criterion.

As mentioned earlier, $\hat{L}_{p}$ is computed on the whole graph. We have $\widehat{L}_{1}\left(X_{i}, X_{j}\right)=\| X_{i}-$ $X_{j} \|$, whereas a power $p>1$ tends to 'select' a path in the graph which is 'close to' the manifold. When $M$ is a $d^{\prime}$-manifold without boundary, and the probability distribution $\mathbb{P}_{X}$ has a density $f_{X}$, Hwang et al. [8] proved that, for $p>1$,

$$
\left|\frac{\widehat{L}_{p}\left(X_{i}, X_{j}\right)}{C_{d^{\prime}, p} n^{(1-p) / d^{\prime}}}-L_{p}\left(X_{i}, X_{j}\right)\right| \stackrel{\text { a.s. }}{\rightarrow} 0, \quad \text { for all }(i, j), n \rightarrow \infty,
$$

where $C_{d^{\prime}, p}$ is a positive constant and $L_{p}(x, y)$ is the geodesic distance on $M$ endowed with the metric $f_{X}^{2(1-p) / d^{\prime}} I_{d^{\prime}}$ (where $I_{d^{\prime}}$ is the identity matrix of size $d^{\prime}$ ). Thus, the estimator $\widehat{L}_{p}$ can only estimate the canonical geodesic distance (that is, $M$ endowed with the identity) when observations are uniformly drawn, while our estimator does not requires such an hypothesis. Moreover, we obtain convergence rates while none are provided for $\widehat{L}$.

We will show, under quite general assumptions on the support $M$, that choosing $r_{n}=$ $d_{h}\left(\mathcal{X}_{n}, M\right)^{2 / 3}$ appears to be convenient (Theorem 2.1). Here and throughout the paper, $d_{h}(A, B)$ denotes the Hausdorff distance between the sets $A$ and $B$; that is,

$$
d_{h}(A, B)=\max \left\{\sup _{a \in A}\left(\inf _{b \in B}\|a-b\|\right), \sup _{b \in B}\left(\inf _{a \in A}\|a-b\|\right)\right\} .
$$

Assuming that $M$ is a $d^{\prime}$-manifold, $d^{\prime} \leq d$, and assuming some regularity for the distribution $\mathbb{P}_{X}$, we show that $d_{h}\left(\mathcal{X}_{n}, M\right)=\mathcal{O}(\ln n / n)^{1 / d^{\prime}}$ everywhere almost surely (e.a.s.), allowing us to find the convergence rate of our estimator when the dimension $d^{\prime}$ is known (Corollary 2.1). When $d^{\prime}$ is unknown, and $M$ is supposed to have no boundary, Corollary 2.2 contains an estimator of $r_{n}$ which allows us to obtain the same convergence rate.

Eventually, we will apply these results to the estimation of the Fréchet expectations, as defined in [13], of the distribution $\mathbb{P}_{X}$ on $M$ (Theorem 2.2).

Using the estimated geodesic distance in place of the Euclidean distance has become frequent in different fields of application in order to take the nonlinearity of the data into account. In [18], the authors proposed to apply the multidimensional scaling (see, for example, [9]) to the array of geodesic distances between points. This idea opened the way to the use of the geodesic distance in dimension reduction (see [5], [10]-[12], and [16]). In [2] and [6], the question of intrinsic dimension estimation using graph-based statistics was studied. In particular, in [6] the authors proposed a generalization of the correlation dimension where the Euclidean distance is 
replaced by the (estimated) geodesic distance. This approach has the advantage that it is less sensitive to the (difficult) question of the choice of parameter (see also [17]). In [13], the author raised the question of the generalization of classical statistical quantities (such as the mean and median) to the case of data supported on Riemannian manifolds.

The paper is organized as follows. In Section 2 we state the general framework, main definitions, and results. In Section 2.1 we present the results concerning the estimation of the geodesic distance on the support $M$ (Theorem 2.1 and Corollaries 2.1 and 2.2), while in Section 2.2 we present the theorem for the Fréchet expectations estimator (Theorem 2.2). Section 3 is devoted to the proofs of the results.

\section{General framework and main results}

\subsection{Estimating geodesic distances}

Let us first start with the definition of our estimator.

Definition 2.1. Let $\mathcal{X}_{n}=\left\{X_{1}, \ldots X_{n}\right\}$ be a set of $n$ i.i.d. random variables with distribution $\mathbb{P}_{X}$ supported on a compact set $M \subset \mathbb{R}^{d}, d \geq 2$. With $r_{n}>0$ being a given number, let $g_{r_{n}}\left(\mathcal{X}_{n}\right)$ be the graph whose edges are the segments $\left[X_{i}, X_{j}\right]$ such that $\left\|X_{i}-X_{j}\right\| \leq r_{n}$.

For $(i, j) \in\{1, \ldots, n\}^{2}$, let, if it exists, $\hat{\gamma}_{r_{n}}\left(X_{i}, X_{j}\right)$ be the shortest path (in the Euclidean norm) connecting $X_{i}$ and $X_{j}$ in $g_{r_{n}}\left(X_{n}\right)$, and $\left|\hat{\gamma}_{n}\left(X_{i}, X_{j}\right)\right|$ its length.

We aim to prove, for a class of convenient compact sets in $\mathbb{R}^{d}$, that $\left|\hat{\gamma}_{r_{n}}\left(X_{i}, X_{j}\right)\right|$ is an estimator of the geodesic distance $\gamma\left(X_{i}, X_{j}\right)$ on $M$, with good convergence properties.

Definition 2.2. Let $M \subset \mathbb{R}^{d}$ be a compact set, $M$ is said to be $K_{M}$-geodesically smooth (GS) for some positive number $K_{M}$ if:

(i) for all $(x, y) \in M^{2}$, there exists a geodesic path $\gamma_{x \rightarrow y}$ of class $\mathcal{C}^{1}$ that links $x$ to $y$;

(ii) there exists a real function $\beta$ such that $\lim _{t \rightarrow 0} \beta(t)=0$ and, for all $(x, y) \in M^{2},\left|\gamma_{x \rightarrow y}\right| \leq$ $\beta(\|x-y\|)$

(iii) let $\Gamma_{x \rightarrow y}:\left[0,\left|\gamma_{x \rightarrow y}\right|\right] \rightarrow \mathbb{R}^{d}$ be the parametrization of $\gamma_{x \rightarrow y}$ such that $\Gamma_{x \rightarrow y}(s)$ is the

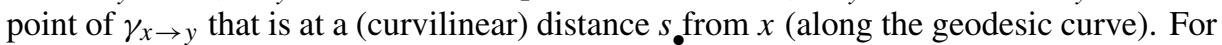
all $(x, y) \in M^{2}$, the gradient of $\Gamma_{x \rightarrow y}$, denoted $\dot{\Gamma}_{x \rightarrow y}$, is $K_{M}$-Lipschitz continuous.

A compact manifold of class $\mathcal{C}^{2}$ with no boundary satisfies the assumptions of Definition 2.2 , but we can build more general examples of such sets (that is, compact sets with $\mathcal{C}^{1}$ geodesic curves which have $K_{M}$-Lipschitz tangent maps). As an example, in Figure 1 we present two examples of GS-sets (sets 1 and 2), and one which is not. Note that the middle panel in Figure 1, while satisfying the GS property, is not a manifold.
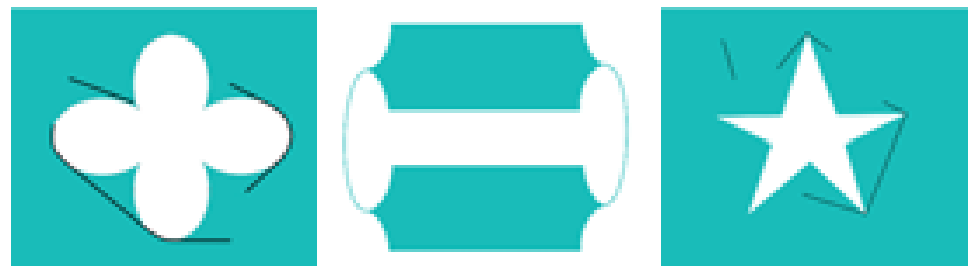

FIgure 1: The sets are the shaded areas. Left: this is GS (with some geodesic curves depicted). Middle: this is also GS (but is not a manifold). Right: this is not GS: some geodesic curves are not smooth enough. 
Theorem 2.1. Let $\hat{\gamma}_{r_{n}}$ be the estimator introduced in Definition 2.1. Assume that there exists a sequence $\rho_{n} \stackrel{\text { a.s. }}{\rightarrow} 0$ such that $\rho_{n} \geq d_{h}\left(\mathcal{X}_{n}, M\right)$ (e.a.s), and let $\left(r_{n}\right)$ be a sequence such that $r_{n}>2 \rho_{n}$ and $\rho_{n} / r_{n} \stackrel{\text { a.s. }}{\rightarrow} 0$. Then

$$
\max _{i, j} \| \hat{\gamma}_{r_{n}}\left(X_{i}, X_{j}\right)|-| \gamma_{X_{i} \rightarrow X_{j}}||=\mathcal{O}\left(\max \left(r_{n}, \frac{\rho_{n}^{2}}{r_{n}^{2}}\right)\right) \text { e.a.s. }
$$

Assuming that $r_{n}>2 \rho_{n}$ ensures the existence of $\left|\hat{\gamma}_{r_{n}}\left(X_{i}, X_{j}\right)\right|$ for all $i$ and $j$. The first part of the proof will clearly illustrate this fact (see Section 3.1).

We can then assume that the sequence $r_{n}=d_{h}\left(\mathcal{X}_{n}, M\right)^{2 / 3}$ is an optimal choice. However, even though it is known that $d_{h}\left(\mathcal{X}_{n}, M\right) \stackrel{\text { a.s. }}{\rightarrow} 0$ (see [4]), the rate of this convergence is unknown in general. Thus, in order to obtain a convergence rate for our estimator, we need to make extra assumptions on the set $M$ and the probability distribution $\mathbb{P}_{X}$.

Definition 2.3. Let $\delta>0$. A probability measure $\mathbb{P}_{X}$ supported on $M \subset \mathbb{R}^{d}$ is said to be $\delta$-standard with respect to a measure $\mu$ if there exists $\lambda>0$ such that $\mathbb{P}_{X}(\mathscr{B}(x, \varepsilon)) \geq$ $\delta \mu(\mathscr{B}(x, \varepsilon))$ for all $x \in M$ and $\varepsilon \in] 0, \lambda]$.

We then have the following result.

Corollary 2.1. Let $M \subset \mathbb{R}^{d}, d \geq 2$, be a $d^{\prime}$-dimensional compact manifold of class $\mathcal{C}^{1}$ satisfying the GS property for some number $K_{M}>0$. Let $\mathbb{P}_{X}$ be a probability distribution on $M$. Assume, for some number $\delta>0$, that $\mathbb{P}_{X}$ is $\delta$-standard with respect to the measure induced on $M$ by the Lebesgue measure in $\mathbb{R}^{d}$.

If the sequence $\left(r_{n}\right)$ in Definition 2.1 is such that

$$
\left(A_{0} \frac{\ln n}{n}\right)^{2 / 3 d^{\prime}} \leq r_{n} \leq\left(A_{1} \frac{\ln n}{n}\right)^{2 / 3 d^{\prime}},
$$

with $A_{0}>0$ and $A_{1}>0$, then

$$
\max _{i, j} \| \hat{\gamma}_{r_{n}}\left(X_{i}, X_{j}\right)|-| \gamma_{X_{i} \rightarrow X_{j}}||=\mathcal{O}\left(\left(\frac{\ln n}{n}\right)^{2 / 3 d^{\prime}}\right) \text { e.a.s. }
$$

As usual when dealing with estimation problems, the sequence of radii $\left(r_{n}\right)$ in the previous theorem remains abstract. In particular, the dimension $d^{\prime}$ of the support is generally unknown. However, making extra assumptions on the support $M$ and the density of the distribution, we can accurately estimate the sequence of radii, with no need for estimating $d^{\prime}$. This last fact is indeed worth emphasizing, since knowledge of the geodesic distance is known to be useful for a good estimation of the dimension of a manifold (see, for example, [6]).

Let $L_{n}=\max _{i}\left(\min _{j \neq i}\left\|X_{i}-X_{j}\right\|\right)$ and let $\theta_{n}$ be the longest edge of the minimal spanning tree of the sample. Up to a rescaling of the data, we can suppose that $\max _{i}\left(\max _{j}\left\|X_{i}-X_{j}\right\|\right) \leq$ 1. Then we have $L_{n}=\max _{i}\left(\min _{j \neq i}\left\|X_{i}-X_{j}\right\|\right) \leq 1$ and $\theta_{n} \leq 1$; hence, $L_{n}^{2 / 3} \geq L_{n}$ and $\theta_{n}^{2 / 3} \geq \theta_{n}$.

Choosing a sequence of radii satisfying $r_{n} \geq \theta_{n}$ ensures the existence of the estimator $\left|\hat{\gamma}_{r_{n}}\left(X_{i}, X_{j}\right)\right|$. Conjecturing that the results of [14] can be generalized to the case of data drawn on a smooth manifold with a density close to the uniform one leads to the choice of $r_{n}=c \cdot \theta_{n}^{2 / 3}$ with $c \geq 1$. If the conjecture is correct, this would guarantee the existence of the estimator and provide optimal convergence rates. More practically, in order to prove a theoretical result, we are led to choosing $r_{n}$ in relation to $L_{n}$. This only ensures the existence of our estimator asymptotically. 
Corollary 2.2. Let $M \in \mathbb{R}^{d}, d \geq 2$, be a $d^{\prime}$-dimensional compact manifold, $d^{\prime}<d$ of class $\mathcal{C}^{2}$ with no boundary, and $\mathbb{P}_{X}$ be a probability distribution on $M$ with continuous probability density $f_{X}$ bounded from below on $M$ by a positive constant $f_{0}$. Then, for any $c>0$, setting $r_{n}=c\left(\max _{i}\left(\min _{j \neq i}\left\|X_{i}-X_{j}\right\|\right)\right)^{2 / 3}$ in Definition 2.1, we have

$$
\max _{i, j} \| \hat{\gamma}_{r_{n}}\left(X_{i}, X_{j}\right)|-| \gamma_{X_{i} \rightarrow X_{j}}||=\mathcal{O}\left(\left(\frac{\ln n}{n}\right)^{2 / 3 d^{\prime}}\right) \text { e.a.s. }
$$

The assumptions of this corollary imply those of Theorem 2.1 ; they allow us to explicitly build a convenient sequence of radii $\left(r_{n}\right)$ only from the sample. To prove Theorem 2.1 we use a result due to Penrose (see [15]) which applies only in the case when $M$ has no boundary. However, numerical simulations on $\mathcal{C}^{2}$ sets with a boundary satisfying the GS assumption lead us to think that the result is also true for such sets.

The question of the choice of the sequence $\left(r_{n}\right)$ remains a difficult subject. In our framework, we propose the following decision rule: first, in the absence of a priori knowledge on the data, and when the support $M$ can have several arcwise connected components (that is, data classes), we choose $r_{n}$ of order $L_{n}^{2 / 3}$. This sequence of radii will converge to 0 and allow us to identify the different classes in the data with optimal convergence rate (although the existence of the estimator is only ensured asymptotically). If we know a priori that the support is arcwise connected, choosing $r_{n}=c \cdot \theta_{n}^{2 / 3}$ with $c \geq 1$ may be a convenient choice, even if the asymptotic properties of the estimator are conjecture-based.

\subsection{Estimating Fréchet expectations}

In this section we assume the set $M$ to be a compact $d^{\prime}$-manifold of class $\mathcal{C}^{2}$. Following the ideas of Pennec (see [13]), we consider the Fréchet expectations of the random variable $X$ (which distribution is supported on $M$ ), that is,

$$
\mathbb{E}_{k}^{\mathrm{Fr}}(X)=\arg \min _{x \in M} \mathbb{E}\left(\left|\gamma_{x \rightarrow X}\right|^{k}\right), \quad k \in \mathbb{N}^{*},
$$

which are generalizations of the expected value for $k=2$ and of the median (or depth) for $k=1$. As pointed out in [13], these expectations are not necessarily unique. For example, if $M$ is a sphere and $\mathbb{P}_{X}$ the uniform distribution, then obviously all the points of $M$ realize the minimum in (2.2) (for any $k \geq 1$ ).

To avoid dealing with such situations, we are going to make the following assumption, considering that $k$ is fixed:

(A) $\Phi(x)=\mathbb{E}\left(\left|\gamma_{x \rightarrow X}\right|^{k}\right)$ admits a unique minimum $x^{*} \in M, \Phi$ is of class $\mathcal{C}^{2}$ in a neighbourhood of $x^{*}$, and $H_{\Phi}\left(x^{*}\right)$ is positive definite,

where $H_{\Phi}$ denotes the hessian matrix of $\Phi$ (that is, $\left.\left(H_{\Phi}\right)_{i, j}=\partial^{2} \Phi /\left(\partial x_{i}, \partial x_{j}\right)\right)$.

Remark 2.1. We must note that $\Phi$ is a continuous function on $M$. Indeed, the triangle and Minkowski inequalities yield $\left|\Phi(x)^{1 / k}-\Phi(y)^{1 / k}\right| \leq\left|\gamma_{x \rightarrow y}\right|$ for any $(x, y) \in M^{2}$. The extra (local) regularity in assumption(A) is required for the sake of simplicity, allowing us to apply basic differential calculus results at the optimal point $x^{*}$.

The first part of this assumption is very strong, but the second part is not. For example, when $d^{\prime}=1$ and $M$ is homeomorphic to a segment, explicit computations show that (A) holds for $k=1$ if and only if $f_{X}\left(x^{*}\right) \neq 0$. For $k=2$, when $M$ is a bounded closed convex set of dimension $d$, the geodesic distance on $M$ coincides with the Euclidean distance, the expectation $\mathbb{E}(X)$ lies in $M$, it minimizes the function $\Phi(x)$, and assumption (A) is satisfied 
(with $H_{\Phi} \equiv 2 I_{d}$ ). This leads us to think that, for $k=2$, this condition is general enough and may hold for a wide class of regular submanifolds of $\mathbb{R}^{d}$.

In this section we study the behavior of the natural estimator of $\mathbb{E}_{k}^{\mathrm{Fr}}(X)$, that is,

$$
\hat{\mathbb{E}}_{k, r_{n}}^{\mathrm{Fr}}\left(X_{n}\right)=\arg \min _{X_{i} \in M} \frac{1}{n} \sum_{j}\left|\hat{\gamma}_{r_{n}}\left(X_{i}, X_{j}\right)\right|^{k} .
$$

Theorem 2.2. Assume that $M \subset \mathbb{R}^{d}, d \geq 2$, is a $d^{\prime}$-dimensional manifold, $d^{\prime}<d$ of class $\mathcal{C}^{2}$ with no boundary, and that $\mathbb{P}_{X}$ is a probability distribution on $M$ with a continuous and bounded from below probability density $f_{X}$. Moreover, suppose that assumption (A) holds. Then, choosing $r_{n}=c\left(\max _{i}\left(\min _{j}\left\|X_{i}-X_{j}\right\|\right)\right)^{2 / 3}$ in the definition of $\hat{\gamma}_{r_{n}}$, we have

$$
\left|\mathbb{E}_{k}^{F r}(X)-\hat{\mathbb{E}}_{k, r_{n}}^{F r}\left(\mathcal{X}_{n}\right)\right|=\mathcal{O}\left(\left(\frac{\ln n}{n}\right)^{\min \left(1 / 4,1 / 3 d^{\prime}\right)}\right) \text { e.a.s. }
$$

\section{Proofs of the results}

Let us start with a result which is a direct consequence of the regularity of the set considered here.

Proposition 3.1. If $M \subset \mathbb{R}^{d}$ is $K_{M}$-geodesically smooth then there exist $r_{M}>0$ and $A_{M}>0$, depending only on $M$, such that

$$
\|x-y\| \leq r_{M} \Longrightarrow\left|\gamma_{x \rightarrow y}\right| \leq\|x-y\|+A_{M}\|x-y\|^{2} \quad \text { for all }(x, y) \in M^{2} .
$$

Proof. Let $(x, y) \in M^{2}$. Consider the parametrization $\Gamma_{x \rightarrow y}$ of the geodesic curve $\gamma_{x \rightarrow y}$ as in Definition 2.2. The map $\dot{\Gamma}$ being $K_{M}$-Lipschitz continuous, for all $t_{0} \in\left[0,\left|\gamma_{x \rightarrow y}\right|\right]$, there exists $\varepsilon_{t_{0}}:\left[0,\left|\gamma_{x \rightarrow y}\right|\right] \rightarrow \mathbb{R}^{d}$ such that

$$
\dot{\Gamma}(t)=\dot{\Gamma}\left(t_{0}\right)+K_{M}\left|t-t_{0}\right| \varepsilon_{t_{0}}(t), \quad\left\|\varepsilon_{t_{0}}(t)\right\| \leq 1 \quad \text { for all } t \in\left[0,\left|\gamma_{x \rightarrow y}\right|\right] .
$$

Thus,

$$
\int_{0}^{\left|\gamma_{x \rightarrow y}\right|} \stackrel{\bullet}{\Gamma}(t) \mathrm{d} t=\int_{0}^{\left|\gamma_{x \rightarrow y}\right|}\left(\dot{\Gamma}\left(t_{0}\right)+K_{M}\left|t-t_{0}\right| \varepsilon_{t_{0}}(t)\right) \mathrm{d} t ;
$$

that is,

$$
y-x=\dot{\Gamma}\left(t_{0}\right)\left|\gamma_{x \rightarrow y}\right|+K_{M} \int_{0}^{\left|\gamma_{x \rightarrow y}\right|}\left|t-t_{0}\right| \varepsilon_{t_{0}}(t) \mathrm{d} t .
$$

Choosing $t_{0}=\frac{1}{2}\left|\gamma_{x \rightarrow y}\right|$, and noting that with the chosen parametrization we have $\left\|\dot{\Gamma}\left(t_{0}\right)\right\|=1$, we obtain

$$
\|x-y\| \geq\left|\gamma_{x \rightarrow y}\right|-\frac{1}{4} K_{M}\left|\gamma_{x \rightarrow y}\right|^{2} \quad \text { for all }(x, y) \in M^{2} .
$$

Now assuming that $\|x-y\| \leq K_{M}^{-1}$, the following alternatives hold:

(i) either $\left|\gamma_{x \rightarrow y}\right| \geq\left(2+2 \sqrt{1-K_{M}\|x-y\|}\right) / K_{M}$,

(ii) or $\left|\gamma_{x \rightarrow y}\right| \leq\left(2-2 \sqrt{1-K_{M}\|x-y\|}\right) / K_{M}$.

For $\|x-y\|$ small enough, the case (i) is impossible due to Definition 2.2(ii). Therefore, there exists $r_{M} \leq K_{M}^{-1}$ such that, for $\|x-y\| \leq r_{M}$, the alternative, case (ii), holds. Making a Taylor expansion of $\|x-y\|$ completes the proof. 


\subsection{Proof of Theorem 2.1}

Let $(i, j) \in\{1, \ldots n\}^{2}, i \neq j$, and let $\gamma_{i j}$ be the geodesic curve between $X_{i}$ and $X_{j}$. Consider a partition $\left\{x_{0}, \ldots, x_{K}\right\}$ of $\gamma_{i j}$ such that

$$
\begin{gathered}
x_{0}=X_{i}, \quad x_{K}=X_{j}, \\
K=\left\lceil\frac{\left|\gamma_{X_{i} \rightarrow X_{j}}\right|}{r_{n}-2 \rho_{n}}\right\rceil, \\
\left|\gamma_{x_{k} \rightarrow x_{k+1}}\right|=\frac{\left|\gamma_{X_{i} \rightarrow X_{j}}\right|}{K},
\end{gathered}
$$

so that

$$
\left|\gamma_{x_{k} \rightarrow x_{k+1}}\right|=r_{n}-2 \rho_{n}, \quad k=0, \ldots, K-2, \quad\left|\gamma_{x_{K-1} \rightarrow x_{K}}\right|<r_{n}-2 \rho_{n} .
$$

We have

$$
\left|\gamma_{X_{i} \rightarrow X_{j}}\right|=\sum_{k=0}^{K-1}\left|\gamma_{x_{k} \rightarrow x_{k+1}}\right| \geq \sum_{k=0}^{K-1}\left\|x_{k}-x_{k+1}\right\| .
$$

From the definition of $\rho_{n}$, for any $k \in\{0, \ldots, K\}$, there exists $i_{k} \in\{1, \ldots, n\}$ such that $\left\|X_{i_{k}}-x_{k}\right\| \leq \rho_{n}$. For the sake of simplicity, denote

$$
Y_{k}=X_{i_{k}}, \quad \varepsilon_{k}=Y_{k}-x_{k}, \quad U_{k}=\frac{x_{k}-x_{k+1}}{\left\|x_{k}-x_{k+1}\right\|}
$$

Recall that

$$
\left\|\varepsilon_{k}\right\| \leq \rho_{n}, \quad k=0, \ldots, K-1 .
$$

For $k \in\{0, \ldots, K-1\}$,

$$
\begin{aligned}
\left\|Y_{k}-Y_{k+1}\right\|^{2} & =\left\|\varepsilon_{k}+\left(x_{k}-x_{k+1}\right)-\varepsilon_{k+1}\right\|^{2} \\
& =\left\|x_{k}-x_{k+1}\right\|^{2}+2\left\langle x_{k}-x_{k+1} \mid \varepsilon_{k}-\varepsilon_{k+1}\right\rangle+\left\|\varepsilon_{k}-\varepsilon_{k+1}\right\|^{2} \\
& =\left\|x_{k}-x_{k+1}\right\|^{2} \times\left(1+2 \frac{\left\langle U_{k} \mid \varepsilon_{k}-\varepsilon_{k+1}\right\rangle}{\left\|x_{k}-x_{k+1}\right\|}+\frac{\left\|\varepsilon_{k}-\varepsilon_{k+1}\right\|^{2}}{\left\|x_{k}-x_{k+1}\right\|^{2}}\right) ;
\end{aligned}
$$

that is, taking the square root of this equality, and noting that $\sqrt{1+t} \leq 1+\frac{1}{2} t, t \geq-1$,

$$
\begin{aligned}
\left\|Y_{k}-Y_{k+1}\right\| & \leq\left\|x_{k}-x_{k+1}\right\| \times\left(1+\frac{\left\langle U_{k} \mid \varepsilon_{k}-\varepsilon_{k+1}\right\rangle}{\left\|x_{k}-x_{k+1}\right\|}+\frac{1}{2} \frac{\left\|\varepsilon_{k}-\varepsilon_{k+1}\right\|^{2}}{\left\|x_{k}-x_{k+1}\right\|^{2}}\right) \\
& \leq\left\|x_{k}-x_{k+1}\right\|+\left\langle U_{k} \mid \varepsilon_{k}-\varepsilon_{k+1}\right\rangle+\frac{1}{2} \frac{\left\|\varepsilon_{k}-\varepsilon_{k+1}\right\|^{2}}{\left\|x_{k}-x_{k+1}\right\|} .
\end{aligned}
$$

In view of (3.1)-(3.4), the length of the last segment, that is, $\left\|x_{K-1}-x_{K}\right\|$, is not bounded from below, hence, we shall treat the cases $k<K-1$ and $k=K-1$ separately. From (3.5), we have

$$
\left|\gamma_{X_{i} \rightarrow X_{j}}\right| \geq\left\|x_{K-1}-x_{K}\right\|+\sum_{k=0}^{K-2}\left\|Y_{k}-Y_{k+1}\right\|-\frac{1}{2} S_{1}-S_{2},
$$

with

$$
S_{1}=\sum_{k=0}^{K-2} \frac{\left\|\varepsilon_{k}-\varepsilon_{k+1}\right\|^{2}}{\left\|x_{k}-x_{k+1}\right\|}, \quad S_{2}=\sum_{k=0}^{K-2}\left\langle U_{k} \mid \varepsilon_{k}-\varepsilon_{k+1}\right\rangle .
$$


We first study $S_{1}$. From (3.4) and Proposition 3.1, we have for $k \in\{0, \ldots, K-2\}$,

$$
r_{n}-2 \rho_{n}-A_{M}\left(r_{n}-2 \rho_{n}\right)^{2} \leq\left\|x_{k}-x_{k+1}\right\| \leq r_{n}-2 \rho_{n} \leq r_{n},
$$

with $A_{M}>0$ depending only on $M$.

Then, for $n$ large enough to have $u_{n}=2 \rho_{n} / r_{n}+A_{M}\left(\left(r_{n}-2 \rho_{n}\right)^{2} / r_{n}\right)<1$ and applying the fact that $1 /(1-u) \leq 1+u$ when $u \in[0,1$, we have, for all $k \in\{0, \ldots, K-2\}$,

$$
\frac{\left\|\varepsilon_{k}-\varepsilon_{k+1}\right\|^{2}}{\left\|x_{k}-x_{k+1}\right\|} \leq \frac{4 \rho_{n}^{2}}{r_{n}-2 \rho_{n}-A_{M}\left(r_{n}-2 \rho_{n}\right)^{2}} \leq \frac{4 \rho_{n}^{2}}{r_{n}}\left(1+\frac{2 \rho_{n}}{r_{n}}+A_{M} \frac{\left(r_{n}-2 \rho_{n}\right)^{2}}{r_{n}}\right) \text {. }
$$

Thus,

$$
\frac{\left\|\varepsilon_{k}-\varepsilon_{k+1}\right\|^{2}}{\left\|x_{k}-x_{k+1}\right\|} \leq \frac{4 \rho_{n}^{2}}{r_{n}}(1+o(1)) .
$$

The definition of $\rho_{n}$ implies that $r_{n}-2 \rho_{n} \sim r_{n}$. Moreover, since the set $M$ is compact and satisfies the GS assumption, $\gamma_{X_{i} \rightarrow X_{j}}$ is uniformly bounded for all $(i, j) \in\{1, \ldots, n\}^{2}$. Hence, there exists a constant $L_{M}>0$ such that

$$
0<K \leq \frac{L_{M}}{r_{n}}
$$

where $K$ is defined by (3.2), and we have

$$
S_{1} \leq L_{M}\left(\frac{4 \rho_{n}^{2}}{r_{n}^{2}}+o\left(\frac{\rho_{n}^{2}}{r_{n}^{2}}\right)\right)
$$

Note that the bound in (3.10) is uniform in $(i, j)$; therefore, the same holds for (3.12).

Now, since the set $M$ is $K_{M}$-geodesically smooth we obtain, reasoning as in the beginning of the proof of Proposition 3.1,

$$
\|\| x_{k+1}-x_{k}\left\|\left(U_{k}-\left(r_{n}-2 \rho_{n}\right)\right) \dot{\Gamma}_{x_{k} \rightarrow x_{k+1}}(0)\right\| \leq \frac{1}{2} K_{M}\left(r_{n}-2 \rho_{n}\right)^{2} .
$$

Thus, applying Proposition 3.1 for $k \in\{0, \ldots, K-2\}$, we have $U_{k}=\dot{\Gamma}_{x_{k} \rightarrow x_{k+1}}(0)+Z_{k}$ with $\left\|Z_{k}\right\| \leq\left(A_{M}+\frac{1}{2} K_{M}\right)\left(r_{n}-2 \rho_{n}\right)$ uniformly in $(i, j)$. Now, noting that $\dot{\Gamma}_{x_{k} \rightarrow x_{k+1}}(0)=$ $\dot{\Gamma}_{x_{0} \rightarrow x_{K}}\left(k\left(r_{n}-2 \rho_{n}\right)\right)$ and due to the Lipshitz continuity of $\dot{\Gamma}$, we have

$$
\left\|U_{k}-U_{k+1}\right\| \leq\left(A_{M}+\frac{3}{2} K_{M}\right)\left(r_{n}-2 \rho_{n}\right) .
$$

We can now write $S_{2}$ as

$$
S_{2}=\sum_{k=1}^{K-2}\left\langle U_{k}-U_{k-1} \mid \varepsilon_{k}\right\rangle+\left\langle U_{0} \mid \varepsilon_{0}\right\rangle-\left\langle U_{K-2} \mid \varepsilon_{K-1}\right\rangle
$$

hence, in view of (3.6), (3.9), (3.11), and (3.13) we have

$$
\left|S_{2}\right| \leq\left(L_{M}\left(A_{M}+\frac{3}{2} K_{M}\right)+2\right) \rho_{n} .
$$

Combining this last inequality with (3.7), (3.8), and (3.12), we obtain the existence of explicit constants $B_{M}>0$ and $C_{M}>0$ depending only on $M$ such that, for large enough $n$ and for all $(i, j)$,

$$
\left|\gamma_{X_{i} \rightarrow X_{j}}\right| \geq \sum_{k=0}^{K-2}\left\|Y_{k}-Y_{k+1}\right\|-B_{M} \rho_{n}-C_{M} \frac{\rho_{n}^{2}}{r_{n}^{2}} .
$$


Thus,

$$
\left|\gamma_{X_{i} \rightarrow X_{j}}\right| \geq \sum_{k=0}^{K-1}\left\|Y_{k}-Y_{k+1}\right\|-\left\|Y_{K-1}-Y_{K}\right\|-B_{M} \rho_{n}-C_{M} \frac{\rho_{n}^{2}}{r_{n}^{2}} .
$$

Recall that, for all $k \in\{0, \ldots, K-1\}$, we have $\left\|Y_{k}-Y_{k+1}\right\|=\left\|\left(x_{k}-x_{k+1}\right)-\left(\varepsilon_{k}-\varepsilon_{k+1}\right)\right\|$; hence, the triangle inequality, (3.4), and (3.6) yield

$$
\left\|Y_{k}-Y_{k+1}\right\| \leq r_{n}, k \in\{0, \ldots, K-1\} .
$$

Applying (3.14) for $k=K-1$, we first obtain

$$
\left|\gamma_{X_{i} \rightarrow X_{j}}\right| \geq \sum_{k=0}^{K-1}\left\|Y_{k}-Y_{k+1}\right\|-r_{n}-B_{M} \rho_{n}-C_{M} \frac{\rho_{n}^{2}}{r_{n}^{2}} .
$$

From (3.14), the path $Y_{0}, \ldots, Y_{K}$ belongs to the graph $g_{r_{n}}\left(\mathcal{X}_{n}\right)$, so we clearly have

$$
\left|\widehat{\gamma}_{r_{n}}\left(X_{i}, X_{j}\right)\right| \leq \sum_{k=0}^{K-1}\left\|Y_{k}-Y_{k+1}\right\|
$$

therefore, since $\rho_{n}=o\left(r_{n}\right)$ and in view of (3.15), we have, for large enough $n$,

$$
\left|\widehat{\gamma}_{r_{n}}\left(X_{i}, X_{j}\right)\right| \leq\left|\gamma_{X_{i} \rightarrow X_{j}}\right|+r_{n}+B_{M} \rho_{n}+C_{M} \frac{\rho_{n}^{2}}{r_{n}^{2}} .
$$

We now prove the following inequality:

$$
\left|\widehat{\gamma}_{r_{n}}\left(X_{i}, X_{j}\right)\right| \geq\left|\gamma_{X_{i} \rightarrow X_{j}}\right|-2 A_{M} L_{M} r_{n} .
$$

For the sake of clarity, we omit the superscripts in Definition 2.1 and denote $Z_{0}=X_{i}, Z_{1}, \ldots$, $Z_{L_{1}}, Z_{L}=X_{j}$ the nodes of the graph $g_{n}^{i, j}$ realizing the path $\widehat{\gamma}_{r_{n}}\left(X_{i}, X_{j}\right)$. Proposition 3.1 yields

$$
\left|\gamma_{X_{i} \rightarrow X_{j}}\right| \leq \sum_{k=0}^{L-1}\left|\gamma_{Z_{k} \rightarrow Z_{k+1}}\right| \leq \sum_{k=0}^{L-1}\left(\left\|Z_{k}-Z_{k+1}\right\|+A_{M}\left\|Z_{k}-Z_{k+1}\right\|^{2}\right) .
$$

Noting, from Definition 2.1, that $\left\|Z_{k}-Z_{k+1}\right\| \leq r_{n}$, we obtain

$$
\left|\widehat{\gamma}_{r_{n}}\left(X_{i}, X_{j}\right)\right| \geq\left|\gamma_{X_{i} \rightarrow X_{j}}\right|-A_{M} L r_{n}^{2} .
$$

We now obtain a bound for the number of nodes $L$ in the path $\widehat{\gamma}_{n}\left(X_{i}, X_{j}\right)$. Necessarily, by construction, we have

$$
\left\|Z_{k}-Z_{k+1}\right\|+\left\|Z_{k+1}-Z_{k+2}\right\|>r_{n}, \quad k=0, \ldots, L-2 .
$$

Indeed, if this was not the case, we would have $\left\|Z_{k}-Z_{k+2}\right\| \leq r_{n}$; hence, the path $\left\{Z_{k}, Z_{k+2}\right\}$ would be shorter in the graph $g_{n}^{i, j}$ than the path $\left\{Z_{k}, Z_{k+1}, Z_{k+2}\right\}$ which is impossible. Therefore, summing up (3.19) for $k \in\{0, \ldots, L-2\}$, we obtain

$$
L r_{n}^{2} \leq 2\left|\widehat{\gamma}_{n}\left(X_{i}, X_{j}\right)\right|+r_{n}^{2}
$$

hence, in view of (3.16), (3.18), and recalling that $\left|\gamma_{X_{i} \rightarrow X_{j}}\right|$ is uniformly bounded, we obtain (3.17). 
This inequality and (3.16) finally imply that

$$
\| \widehat{\gamma}_{r_{n}}\left(X_{i}, X_{j}\right)|-| \gamma_{X_{i} \rightarrow X_{j}}|| \leq D_{M} \max \left(\frac{\rho_{n}^{2}}{r_{n}^{2}}, r_{n}\right),
$$

where the constant $D_{M}>0$ only depends on the manifold $M$. This yields the estimate (2.1) and concludes the proof.

\subsection{Proof of Corollary 2.1}

Reasoning as in [1], since $M$ is of class $\mathcal{C}^{1}$, we can cover $M$ with $v_{n} \leq C n$ (with $C>0$ ) deterministic balls of radius $\varepsilon_{n}=(1 / n)^{1 / d^{\prime}}$ with centers $x_{i} \in M, i \in\left\{1, \ldots, v_{n}\right\}$. Let $\omega_{d^{\prime}}$ be the volume of the $d^{\prime}$-dimensional unit ball. Recall that $\mathbb{P}_{X}$ is $\delta$-standard with respect to the $d^{\prime}$-dimensional measure. We then classically have, for all $a>0$,

$$
\begin{aligned}
& \mathbb{P}_{X}\left(d_{h}\left(\mathcal{X}_{n}, M\right) \geq\left(\frac{2 a}{\delta \omega_{d^{\prime}}} \frac{\ln n}{n}\right)^{1 / d^{\prime}}\right) \\
& \quad=\mathbb{P}_{X}\left(\text { there exists } x \in M ; \mathcal{B}\left(x,\left(\frac{2 a}{\delta \omega_{d^{\prime}}} \frac{\ln n}{n}\right)^{1 / d^{\prime}}\right) \cap \mathcal{X}_{n}=\varnothing\right) .
\end{aligned}
$$

The triangle inequality thus implies

$$
\begin{aligned}
& \mathbb{P}_{X}\left(d_{h}\left(\mathcal{X}_{n}, M\right) \geq\left(\frac{2 a}{\delta \omega_{d^{\prime}}} \frac{\ln n}{n}\right)^{1 / d^{\prime}}\right) \\
& \quad \leq \mathbb{P}_{X}\left(\text { there exists } i ; \mathscr{B}\left(x_{i},\left(\frac{2 a}{\delta \omega_{d^{\prime}}} \frac{\ln n}{n}\right)^{1 / d^{\prime}}-\varepsilon_{n}\right) \cap \mathcal{X}_{n}=\varnothing\right) .
\end{aligned}
$$

Since $\mathbb{P}_{X}$ is standard, we have, for large enough $n,\left(\left(2 a / \delta \omega_{d^{\prime}}\right)(\ln n / n)\right)^{1 / d^{\prime}}<\lambda$. Thus,

$$
\mathbb{P}_{X}\left(d_{h}\left(\mathcal{X}_{n}, M\right) \geq\left(\frac{2 a}{\delta \omega_{d^{\prime}}} \frac{\ln n}{n}\right)^{1 / d^{\prime}}\right) \leq v_{n}\left(1-\delta \omega_{d^{\prime}}\left(\left(\frac{2 a}{\delta \omega_{d^{\prime}}} \frac{\ln n}{n}\right)^{1 / d^{\prime}}-\varepsilon_{n}\right)^{d^{\prime}}\right)^{n}
$$

A Taylor expansion of the right-hand side of the above inequality yields

$$
\mathbb{P}_{X}\left(d_{h}\left(X_{n}, M\right) \geq\left(\frac{2 a}{\delta \omega_{d^{\prime}}} \frac{\ln n}{n}\right)^{1 / d^{\prime}}\right) \leq C n^{1-2 a+o(1)} \quad \text { for any } a>0 .
$$

Applying the Borel-Cantelli lemma, we deduce that, for any $a>1$,

$$
d_{h}\left(\mathcal{X}_{n}, M\right) \leq\left(\frac{2 a}{\delta \omega_{d^{\prime}}} \frac{\ln n}{n}\right)^{1 / d^{\prime}} \quad \text { e.a.s. }
$$

Eventually, applying Theorem 2.1 completes the proof.

\subsection{Proof of Corollary 2.2}

Let

$$
t_{n}=\max _{i}\left(\min _{j}\left\|X_{i}-X_{j}\right\|\right) .
$$

Applying [15, Theorem 5.1, p. 958], we have

$$
\frac{n \omega_{d^{\prime}} t_{n}^{d^{\prime}}}{\ln n} \stackrel{\text { a.s. }}{\rightarrow} f_{0}^{-1}
$$


Therefore, we easily deduce that

$$
\left(\frac{1}{2 f_{0} \omega_{d^{\prime}}} \frac{\ln n}{n}\right)^{1 / d^{\prime}} \leq t_{n} \leq\left(\frac{2}{f_{0} \omega_{d^{\prime}}} \frac{\ln n}{n}\right)^{1 / d^{\prime}} \text { e.a.s. }
$$

Since we have $r_{n}=\left(c t_{n}\right)^{2 / 3}$, the assumptions of Corollary 2.1 are fulfilled, which allows to conclude the proof.

\subsection{Proof of Theorem 2.2}

In view of assumption (A) and (2.3), we introduce the estimators

$$
\begin{aligned}
& \bar{\Phi}(x)=\frac{1}{n} \sum_{i}\left(\left|\gamma_{x \rightarrow X_{i}}\right|^{k}\right), \\
& \hat{\Phi}(x)=\frac{1}{n} \sum_{i}\left(\left|\hat{\gamma}_{r_{n}}\left(x, X_{i}\right)\right|^{k}\right) .
\end{aligned}
$$

First, we prove that there exists a deterministic constant $D>0$ such that

$$
\max _{i}\left|\hat{\Phi}\left(X_{i}\right)-\Phi\left(X_{i}\right)\right|=D\left(\frac{\ln n}{n}\right)^{\min \left\{2 / 3 d^{\prime}, 1 / 2\right\}} \text { e.a.s }
$$

Indeed, the manifold $M$ being compact, one can apply the Hoeffding inequality and obtain

$$
\mathbb{P}_{X}\left(|\bar{\Phi}(x)-\Phi(x)| \geq \varepsilon_{n}\right) \leq 2 \exp \left(-\frac{2 n \varepsilon_{n}^{2}}{L^{2 k}}\right) \text { for all } x \in M,
$$

where $L>0$ is the constant introduced in the proof of Theorem 2.1. Hence,

$$
\left.\mathbb{P}_{X} \text { (there exists } i \in\{1, \ldots, n\} ;\left|\bar{\Phi}\left(X_{i}\right)-\Phi\left(X_{i}\right)\right| \geq \varepsilon_{n}\right) \leq 2 n \exp \left(-\frac{2 n \varepsilon_{n}^{2}}{L^{2 k}}\right) .
$$

Setting $\varepsilon_{n}=\sqrt{2} L^{k} \sqrt{\ln n / n}$ in this last inequality yields

$$
\mathbb{P}_{X}\left(\max _{i}\left|\bar{\Phi}\left(X_{i}\right)-\Phi\left(X_{i}\right)\right| \geq \varepsilon_{n}\right) \leq 2 n^{-3}
$$

so that the Borel-Cantelli lemma allows to conclude that

$$
\max _{i}\left|\bar{\Phi}\left(X_{i}\right)-\Phi\left(X_{i}\right)\right|=\mathcal{O}\left(\frac{\ln n}{n}\right)^{1 / 2} \text { e.a.s. }
$$

Now, noting that the assumptions of Corollary 2.2 are fulfilled, we have

$$
\max _{i}\left|\hat{\Phi}\left(X_{i}\right)-\bar{\Phi}\left(X_{i}\right)\right|=\mathcal{O}\left(\frac{\ln n}{n}\right)^{2 / 3 d^{\prime}} \quad \text { e.a.s. }
$$

Combining this with (3.22), we obtain (3.21).

Next, since $\Phi$ is continuous on the compact set $M$, and in view of assumption (A), the gradient of $\Phi$ vanishes at the (unique) minimum point $x^{*}$; hence, there exist $r_{0}>0, c_{0}>0, c_{1}>0$, and $\varepsilon_{0}$ such that

$$
\begin{gathered}
\Phi(x) \geq \Phi\left(x^{*}\right)+\varepsilon_{0} \quad \text { for all } x \in M \cap B^{c}, \\
c_{0}\left\|x-x^{*}\right\|^{2} \leq \Phi(x)-\Phi\left(x^{*}\right) \leq c_{1}\left\|x-x^{*}\right\|^{2} \text { for all } x \in M \cap \bar{B},
\end{gathered}
$$


where $B=\mathscr{B}\left(x^{*}, r_{0}\right)$ is the open ball in $\mathbb{R}^{d}$ of center $x^{*}$ and radius $r_{0}$. The second inequality holds due to the positiveness of the Hessian matrix $H_{\Phi}\left(x^{*}\right)$.

Now, since the assumptions of Corollary 2.2 are satisfied, there exists $C>0$ such that $d_{h}\left(\mathcal{X}_{n}, M\right) \leq C(\ln n / n)^{1 / d^{\prime}}$. Thus, e.a.s., there exists $i_{0} \in\{1, \ldots, n\}$ such that

$$
\left\|X_{i_{0}}-x^{*}\right\| \leq C\left(\frac{\ln n}{n}\right)^{1 / d^{\prime}}
$$

For large enough $n$, we have $r_{0}>C(\ln n / n)^{1 / d^{\prime}}$; hence, in view of (3.24), $X_{i_{0}}$ satisfies

$$
\Phi\left(X_{i_{0}}\right) \leq \Phi\left(x^{*}\right)+c_{1} C^{2}\left(\frac{\ln n}{n}\right)^{2 / d^{\prime}},
$$

and from (3.21),

$$
\hat{\Phi}\left(X_{i_{0}}\right) \leq \Phi\left(x^{*}\right)+c_{1} C^{2}\left(\frac{\ln n}{n}\right)^{2 / d^{\prime}}+D\left(\frac{\ln n}{n}\right)^{2 \alpha}
$$

with

$$
\alpha=\min \left\{\frac{1}{3 d^{\prime}}, \frac{1}{4}\right\} ;
$$

that is, for large enough $n$, there exists $i_{0} \in\{1, \ldots, n\}$ such that

$$
\hat{\Phi}\left(X_{i_{0}}\right) \leq \Phi\left(x^{*}\right)+2 D\left(\frac{\ln n}{n}\right)^{2 \alpha} .
$$

Assume now that $n$ is large enough so that $\varepsilon_{0}>4 D(\ln n / n)^{2 \alpha}$. For any $i \in\{1, \ldots, n\}$ such that

$$
\left\|X_{i}-x^{*}\right\| \geq \frac{2 D}{\sqrt{c_{0}}}\left(\frac{\ln n}{n}\right)^{\alpha},
$$

in view of (3.23) and (3.24), this point satisfies

$$
\Phi\left(X_{i}\right) \geq \Phi\left(x^{*}\right)+4 D\left(\frac{\ln n}{n}\right)^{2 \alpha} .
$$

Thus, in view of (3.21), we have

$$
\left\|X_{i}-x^{*}\right\| \geq \frac{2 D}{\sqrt{c_{0}}}\left(\frac{\ln n}{n}\right)^{\alpha} \Longrightarrow \hat{\Phi}\left(X_{i}\right) \geq \Phi\left(x^{*}\right)+3 D\left(\frac{\ln n}{n}\right)^{2 \alpha} .
$$

Finally, let $i^{*} \in\{1, \ldots, n\}$ such that $X_{i^{*}}$ realizes the minimum (2.3). From (3.20) and (3.25), it is clear that

$$
\hat{\Phi}\left(X_{i^{*}}\right) \leq \Phi\left(x^{*}\right)+2 D\left(\frac{\ln n}{n}\right)^{2 \alpha},
$$

and (3.26) allows us to conclude the proof. 


\section{References}

[1] Baillo, A., Cuevas, A. and Justel, A. (2000). Set estimation and nonparametric detection. Canad. J. Statist. 28, 765-782.

[2] Brito, M. R., Quiroz, A. J. AND Yukich, J. E. (2013). Intrinsic dimension identification via graph-theoretic methods. J. Multivariate Anal. 116, 263-277.

[3] Cormen, T. H., Leiserson, C. E., Rivest, R. L. and Stein, C. (2001). Introduction to Algorithms, 3rd edn. MIT Press, Cambridge, MA.

[4] Cuevas, A. and Rodríguez-Casal, A. (2004). On boundary estimation. Adv. Appl. Prob. 36, 340-354.

[5] Demartines, P. And Herault, J. (1997). Curvilinear component analysis: a self-organizing neural network for nonlinear mapping of data sets. IEEE Trans. Neural Networks 8, 148-154.

[6] Granata, D. and Carnevale, V. (2016). Accurate estimation of the intrinsic dimension using graph distances: unraveling the geometric complexity of datasets. Sci. Rep. 6, 31377.

[7] Howard, C. D. And Newman, C. M. (2001). Geodesics and spanning trees for Euclidean first-passage percolation. Ann. Prob. 29, 577-623.

[8] Hwang, S. J., Damelin, S. B. And Hero III, A. O. (2016). Shortest path through random points. Ann. Appl. Prob. 26, 2791-2823.

[9] KrusKal, J. B. (1964). Multidimensional scaling by optimizing goodness of fit to a nonmetric hypothesis. Psychometrika 29, 1-27.

[10] Lee, J. A., Lendasse, A. And Verleyzen, M. (2004). Nonlinear projection with curvilinear distances: isomap versus curvilinear distance analysis. Neurocomputing 57, 49-76.

[11] Lennon, M., Mercier, G., Mouchot, M. C. and Hubert-Moy, L. (2001). Curvilinear component analysis for nonlinear dimensionality reduction of hyperspectral images. Proc. SPIE Image and Signal Process. for Remote Sens. VII, 4541, 157-168.

[12] Nilsson, J., Fioretos, T., Höglund, M. And Fontes, M. (2004). Approximate geodesic distances reveal biologically relevant structures in microarray data. Bioinformatics 20, 874-880.

[13] Pennec, X. (2006). Intrinsic statistics on Riemannian manifolds: basic tools for geometric measurements. J. Math. Imaging Vision 25, 127-154.

[14] Penrose, M. D. (1997). The longest edge of the random minimal spanning tree. Ann. Appl. Prob. 7, $340-361$.

[15] Penrose, M. D. (1999). A strong law for the largest nearest-neighbour link between random points. J. London Math. Soc. 60, 951-960.

[16] SaUl, L. K. AND Roweis, S. T. (2003). Think globally, fit locally: unsupervised learning of low dimensional manifolds. J. Mach. Learn. Res. 4, 119-155.

[17] Takens, F. (1985). On the numerical determination of the dimension of an attractor. In Dynamical Systems and Bifurcations (Lectures Notes Math. 1125), Springer, Berlin, pp. 99-106.

[18] Tenenbaum, J. B., De Silva, V. And Langford, J. C. (2000). A global geometric framework for nonlinear dimensionality a global geometric framework for nonlinear dimensionality reduction. Science 290, 2319-2323. 